\title{
Scalar fluctuations of the scalar metric during inflation from a non-perturbative 5D large-scale repulsive gravity model
}

\author{
${ }^{1}$ José Edgar Madriz Aguilar, ${ }^{1}$ Luz M. Reyes , ${ }^{1}$ Claudia Moreno and ${ }^{2,3}$ Mauricio Bellini, \\ 1 Departamento de Matemáticas, \\ Centro Universitario de Ciencias Exactas e ingenierías (CUCEI), \\ Universidad de Guadalajara (UdG), \\ Av. Revolución 1500 S.R. 44430, \\ Guadalajara, Jalisco, México. \\ E-mail: edgar.madriz@red.cucei.udg.mx, \\ luzmarinareyes@gmail.com, claudia.moreno@cucei.udg.mx \\ and \\ 2 Departamento de Física, \\ Facultad de Ciencias Exactas y Naturales, \\ Universidad Nacional de Mar del Plata (UNMdP), \\ Funes 3350, C.P. 7600, Mar del Plata, Argentina \\ E-mail: mbellini@mdp.edu.ar \\ 3 Instituto de Investigaciones Físicas de Mar del Plata (IFIMAR)- Consejo \\ Nacional de Investigaciones Científicas y Técnicas (CONICET) Argentina.
}

\begin{abstract}
We develop a non-perturbative formalism for scalar metric fluctuations from a 5D extended version of General Relativity in vacuum. In this work we concentrate our efforts on calculations valid on large cosmological scales, which are the dominant during the inflationary phase of the universe. The resulting metric on this limit case is obtained after implementing a planar coordinate transformation on a 5D Ricci-flat metric solution. We calculate the spectrum of these fluctuations on an effective 4D Schwarzschil-de Sitter spacetime on cosmological scales, which is obtained after make a static foliation on the noncompact extra coordinate. Our results show how the squared metric fluctuations of the primordial universe become scale invariant with the inflationary expansion.
\end{abstract}

Keywords: scalar metric fluctuations, cosmological inflation, 5D non-compact Kaluza-Klein gravity, Schwarzschildde-Sitter metric.

\section{INTRODUCTION}

In the last years the study of the early universe has raised the interest of cosmologists due to some new issues on this topic as new inflationary cosmologies, quantum gravity, etc. Currently Inflation is a well grounded theory having a experimental status, that has a good interface with high-energy physics and provides various approaches to topical problems of the fundamental physics, specifically to the problem of divergence in a quantum theory or the singularity problem in the General Theory of Relativity. Furthermore, in some way it leads to the problem of quantum-gravitational effects and their adequate description. These reasons, among others, make the investigation of the Early Universe of particular importance. Inflationary cosmology is one of the paradigms that will be confirmed with new observational data coming from the PLANCK satellite. Inflation has become the standard paradigm for explaining the homogeneity and the isotropy of our observed Universe $[1,2]$. During this epoch the energy density of the Universe was dominated by some scalar field (the inflaton), with negligible kinetic energy density, in such a way that its corresponding vacuum energy density is responsible for the exponential growth of the scale factor of the universe. Along this phase a small and smooth region of the order of size of the Hubble radius, grew so large that it easily encompassed the comoving volume of the entire presently observed Universe, and consequently the observable universe become so homogeneous and isotropic, spatially. Moreover, it is now clear that the structure in the Universe has its origin primarily from an almost scale-invariant super-horizon curvature perturbation. Unfortunately, there are plenty of inflationary models the majority of them in good concordance with observations, but none free of problems, as for example the transplanckian problem, the hierachy problem, etc [3]. This has lead cosmologists to look for some new theoretical alternatives [4]. In particular resort to extra dimensions has been of some success.

The extension of the $4 \mathrm{D}$ spacetime to $N(\geq 5) D$ manifolds is the preferred route to an unification of the interactions of particle physics with gravity. The basic extension to 5D describes a spin- 2 graviton, a spin- 1 photon and a spin-0 scalaron, which can be related respectively to the Einstein, Maxwell and Higgs fields [5]. In the cosmological context the extra dimension is already known to be of great importance. There is a class of 5D cosmological 
models which reduce to the usual four dimensional ones, on hypersurfaces defined by establishing a foliation on the fifth extra coordinate. In these models the matter is explained as a consequence of the geometry in five dimensions.

The idea that the equation of state for matter is $\omega_{m}=-1$ (and not $\omega_{m}=0$ ) was previously explored in some works using some ideas of the Space-Time-Matter (STM) theory, from gravitational [6] and cosmological [7] points of view. The origin of gravitational waves have been also considered for a large-scale repulsive gravitational field from a $5 \mathrm{D}$ vacuum [8]. In this work we develop a nonperturbative formalism for scalar metric fluctuations from a 5D extended Schwarzschild-de Sitter (SdS) cosmological metric. It can be seen that on cosmological scales gravity becomes repulsive and these fluctuations behave as a longitudinal gauge [9]. Finally, we study the spectrum for the squared fluctuations of the metric.

\section{THE 5D FIELD EQUATIONS}

On the coordinate chart $\{T, R, \theta, \phi, \psi\}$, let us consider the $5 \mathrm{D}$ line element

$$
d S_{5}^{2}=\left(\frac{\psi}{\psi_{0}}\right)^{2}\left[c^{2} f(R) d T^{2}-\frac{d R^{2}}{f(R)}-R^{2}\left(d \theta^{2}+\sin ^{2} \theta d \phi^{2}\right)\right]-d \psi^{2}
$$

that is describing a Ricci-flat metric [10]. Here, $f(R)=1-\left[\left(2 G \zeta \psi_{0} /\left(R c^{2}\right)\right]-\left(R / \psi_{0}\right)^{2}\right.$ is a dimensionless metric function, $\psi$ is the spacelike and non-compact fifth extra coordinate ${ }^{1}$. This metric is an extension to $5 \mathrm{D}$ spaces of the $4 \mathrm{D}$ SdS metric. $T$ is a time-like coordinate, $c$ is denoting the speed of light, $R, \theta, \phi$ are the usual spherical polar coordinates, $\psi_{0}$ is an arbitrary constant with length units and the constant parameter $\zeta$ has units of $($ mass $)(\text { length })^{-1}$. The metric (1) is static, however, it can be written on a dynamical coordinate chart $\{t, r, \theta, \phi, \psi\}$ by implementing the planar coordinate transformation [11]

$$
R=\operatorname{ar}\left[1+\frac{G \zeta \psi_{0}}{2 a r}\right]^{2}, \quad T=t+H \int^{r} d R \frac{R}{f(R)}\left(1-\frac{2 G \zeta \psi_{0}}{R}\right)^{-1 / 2},
$$

$a(t)=a_{0} e^{t / \psi_{0}}$ being the scale factor. After doing so, the line element (1) may be expressed in terms of the conformal time $\tau$ in the form

$$
d S_{5}^{2}=\left(\frac{\psi}{\psi_{0}}\right)^{2}\left[F(\tau, r) d \tau^{2}-J(\tau, r)\left(d r^{2}+r^{2}\left(d \theta^{2}+\sin ^{2} \theta d \phi^{2}\right)\right)\right]-d \psi^{2},
$$

where the metric functions $F(\tau, r)$ and $J(\tau, r)$ are given by

$$
F(\tau, r)=a^{2}(\tau)\left[1-\frac{G \zeta \psi_{0}}{2 a(\tau) r}\right]^{2}\left[1+\frac{G \zeta \psi_{0}}{2 a(\tau) r}\right]^{-2}, \quad J(\tau, r)=a^{2}(\tau)\left[1+\frac{G \zeta \psi_{0}}{2 a(\tau) r}\right]^{4}
$$

with $d \tau=a^{-1}(\tau) d t$ and $a(\tau)=-\psi_{0} / \tau$, so that the constant Hubble parameter satisfies

$$
H=\frac{1}{\psi_{0}}=a^{-2} \frac{d a}{d \tau}
$$

Notice that the metric in (3) is no more Ricci-flat. As was shown in [10], for certain values of $\zeta$ and $\psi_{0}$, the metric in (1) has two natural horizons. The inner one is the analogous to the Schwarzschild horizon and the external one is the analogous to the Hubble Horizon. In the metric in (3) these horizons may be written in the new dynamical coordinate chart.

The non-perturbative metric fluctuations of the background (3), are introduced through the perturbed line element

$$
\left.d S_{5}^{2}\right|_{\text {pert }}=\left(\frac{\psi}{\psi_{0}}\right)^{2}\left[F(\tau, r) e^{2 \Phi} d \tau^{2}-J(\tau, r) e^{-2 \Phi}\left(d r^{2}+r^{2}\left(d \theta^{2}+\sin ^{2} \theta d \phi^{2}\right)\right)\right]-d \psi^{2}
$$

\footnotetext{
${ }^{1}$ In our notation conventions henceforth, latin indices $a, b=$ run from 0 to 4 , whereas the rest of latin indices $i, j, n, l, \ldots=$ run from 1 to 3.
} 
where $\Phi(\tau, r, \theta, \phi, \psi)$ is the metric function that describes the gauge invariant scalar metric fluctuations. In cartesian coordinates this perturbed line element reads

$$
\left.d S_{5}^{2}\right|_{p e r t}=\left(\frac{\psi}{\psi_{0}}\right)^{2}\left[F(\tau, x, y, z) e^{2 \Phi} d \tau^{2}-J(\tau, x, y, z) e^{-2 \Phi} \delta_{i j} d x^{i} d x^{j}\right]-d \psi^{2}
$$

being now $\Phi=\Phi(\tau, x, y, z, \psi)$. For simplicity, we will use (7) to obtain the Einstein field equations in 5D.

Now let us to consider a non-massive scalar field $\varphi\left(x^{a}\right)$ on the $5 \mathrm{D}$ spacetime $(7)$. Its dynamics can be derived from the action

$$
{ }^{(5)} \mathcal{S}=\int \sqrt{g_{5}}\left[\frac{{ }^{(5)} R}{2 \kappa_{5}}-\frac{1}{2} g^{a b} \varphi_{, a} \varphi, b\right] d^{4} x d \psi
$$

where ${ }^{(5)} R$ is the Ricci scalar, $g_{5}$ is the determinant of the metric (7) and $\kappa_{5}$ is the $5 \mathrm{D}$ gravitational coupling constant. As the scalar field $\varphi$ is purely kinetic there are no interactions of this field with its environment in 5D, however in 4D this is not the case. Clearly, the energy-momentum tensor derived from (8) reads

$$
{ }^{(5)} T_{a b}=\varphi_{, a} \varphi, b-\frac{1}{2} g_{a b} \varphi, c \varphi^{, c}
$$

which is obviously symmetric. The dynamics of the scalar field $\varphi$ derived from the action (8) on the perturbed metric (7) is given by

$$
\ddot{\varphi}+\left[\left(\frac{3}{2} \frac{\dot{J}}{J}-\frac{1}{2} \frac{\dot{F}}{F}\right)-4 \dot{\Phi}\right] \dot{\varphi}-\sqrt{\frac{F}{J^{3}}} e^{4 \Phi} \vec{\nabla}(\sqrt{F J}) \cdot \vec{\nabla} \varphi-\frac{F}{J} e^{4 \Phi} \nabla^{2} \varphi+\left(\frac{\psi}{\psi_{0}}\right)^{2} F e^{2 \Phi}\left[\left(2 \stackrel{\star}{\Phi}^{2}-\frac{4}{\psi}\right) \stackrel{\star}{\varphi}-\stackrel{\star \star}{\varphi}\right]=0
$$

where we denote $(\vec{\nabla} \Phi)^{2} \equiv \vec{\nabla} \Phi \cdot \vec{\nabla} \Phi$, the $\operatorname{dot}(\cdot)$ denotes partial derivative with respect to the conformal time $\tau$ and the star $(\star)$ is denoting partial derivative with respect to the extra coordinate $\psi$. The diagonal components of the 5D field equations ${ }^{(5)} G_{a b}=k_{5}{ }^{(5)} T_{a b}$, on the background (7), have the form

$$
\begin{aligned}
& -\frac{3}{4}\left(\frac{\dot{J}}{J}\right)^{2}+\frac{3 \dot{J}}{J} \dot{\Phi}-3 \dot{\Phi}^{2}+\left[\frac{3 F}{\psi_{0}^{2}}\right. \\
& \left.\left.-3 F\left(\frac{\psi}{\psi_{0}}\right)^{2} \stackrel{\star \star \star}{\Phi}-2 \stackrel{\star^{2}}{\Phi}\right)-\frac{12 F \psi}{\psi_{0}^{2}} \stackrel{\star}{\Phi}\right] e^{2 \Phi}-\left[\frac{3 F}{4 J^{3}}(\vec{\nabla} J)^{2}+\frac{F}{J^{2}}(\vec{\nabla} J \cdot \vec{\nabla} \Phi)-\frac{F}{J}(\vec{\nabla} \Phi)^{2}+\frac{2 F}{J} \nabla^{2} \Phi-\frac{F}{J^{2}} \nabla^{2} J\right] e^{4 \Phi} \\
& =-\kappa_{5}\left[\frac{1}{2} \dot{\varphi}^{2}+\frac{1}{2}\left(\frac{F}{J}\right) e^{4 \Phi}(\vec{\nabla} \varphi)^{2}+\frac{1}{2}\left(\frac{\psi}{\psi_{0}}\right)^{2} F e^{2 \Phi} \stackrel{\star}{\varphi}^{2}\right], \\
& \frac{3}{4}\left(\frac{\vec{\nabla} J}{J}\right)^{2}+\frac{1}{2}\left(\frac{\vec{\nabla} F}{F}\right)^{2}-\left(\frac{\vec{\nabla} F \cdot \vec{\nabla} J}{2 F J}\right)-\frac{\nabla^{2} J}{J}-\frac{\nabla^{2} F}{F}-\frac{\vec{\nabla} F \cdot \vec{\nabla} \Phi}{F}-(\vec{\nabla} \Phi)^{2} \\
& +\left[-\left(\frac{9 J}{\psi_{0}^{2}}\right)-6 J\left(\frac{\psi}{\psi_{0}}\right)^{2} \stackrel{\star^{2}}{\Phi}+\frac{12 J \psi}{\psi_{0}^{2}} \stackrel{\star}{\Phi}+3 J\left(\frac{\psi}{\psi_{0}}\right)^{2} \stackrel{\star \star}{\Phi}\right] e^{-2 \Phi} \\
& +\left[\left(\frac{3 J \dot{F}}{F^{2}}-\frac{12 \dot{J}}{F}\right) \dot{\Phi}-\frac{3 \dot{J}^{2}}{4 F J}-\frac{3 \dot{F} \dot{J}}{2 F^{2}}+\frac{3 \ddot{J}}{F}+\frac{15 J}{F} \dot{\Phi}^{2}-\frac{6 J}{F} \ddot{\Phi}\right] e^{-4 \Phi} \\
& =-\kappa_{5}\left[\frac{3 J}{2 F} e^{-4 \Phi} \dot{\varphi}^{2}-\frac{1}{2}(\vec{\nabla} \varphi)^{2}-\frac{3 J}{2}\left(\frac{\psi}{\psi_{0}}\right)^{2} e^{-2 \Phi} \stackrel{\star}{\varphi}^{2}\right] \text {, } \\
& -\frac{6}{\psi^{2}}+\frac{6}{\psi} \stackrel{\star}{\Phi}+\left(\frac{\psi_{0}}{\psi}\right)^{2}\left[\frac{(\vec{\nabla} F)^{2}}{4 J F^{2}}+\frac{3(\vec{\nabla} J)^{2}}{4 J^{3}}-\frac{\vec{\nabla} F \cdot \vec{\nabla} J}{4 F J^{2}}-\frac{\nabla^{2} J}{J^{2}}-\frac{\nabla^{2} F}{2 F J}-\frac{1}{2}\left(\frac{\vec{\nabla} F}{F J}-\frac{\vec{\nabla} J}{J^{2}}\right) \cdot \vec{\nabla} \Phi-\frac{(\vec{\nabla} \Phi)^{2}}{J}+\frac{\nabla^{2} \Phi}{J}\right] e^{2 \Phi} \\
& +\left(\frac{\psi_{0}}{\psi}\right)^{2}\left[-\frac{3}{4} \frac{\dot{F} \dot{J}}{F^{2} J}+\frac{3}{2} \frac{\ddot{J}}{F J}-\frac{3}{2}\left(\frac{5 \dot{J}}{F J}-\frac{\dot{F}}{F^{2}}\right) \dot{\Phi}+\frac{9}{F} \dot{\Phi}^{2}-\frac{3}{F} \ddot{\Phi}\right] e^{-2 \Phi} \\
& =-\kappa_{5}\left[\frac{1}{2 F}\left(\frac{\psi}{\psi_{0}}\right)^{-2} e^{-2 \Phi} \dot{\varphi}^{2}-\frac{1}{2 J}\left(\frac{\psi}{\psi_{0}}\right)^{-2} e^{2 \Phi}(\vec{\nabla} \varphi)^{2}+\frac{1}{2} \stackrel{\star}{\varphi}^{2}\right] \text {, }
\end{aligned}
$$


while the non-diagonal 5D Einstein equations are given by

$$
\begin{aligned}
& \frac{\dot{J}_{, i}}{J}-\frac{1}{2} \frac{\dot{J} F_{, i}}{J F}-\frac{\dot{J} J_{, i}}{J^{2}}+2 \Phi_{, i} \dot{\Phi}-\left(\frac{\dot{J}}{J}\right) \Phi_{, i}-2 \dot{\Phi}_{, i}+\left(\frac{F_{, i}}{F}\right) \dot{\Phi}=-\kappa_{5} \dot{\varphi} \varphi_{, i}, \\
& 6 \stackrel{\star}{\Phi} \dot{\Phi}-3\left(\frac{\dot{J}}{J}\right) \stackrel{\star}{\Phi}-3 \stackrel{\star \star}{\Phi}=-\kappa_{5} \dot{\varphi} \stackrel{\star}{\varphi}, \\
& 2 \Phi_{, i} \stackrel{\star}{\Phi}+\left(\frac{F_{, i}}{F}\right) \stackrel{\star}{\Phi}-\stackrel{\star}{\Phi}, i=-\kappa_{5} \varphi, \stackrel{\star}{\varphi}, \\
& 2 \Phi_{, i} \Phi_{, j}+\frac{F_{, i}}{F} \Phi_{, j}+\frac{F_{, j}}{F} \Phi_{, i}+\frac{1}{2} \frac{F_{, i j}}{F}-\frac{1}{4} \frac{F_{, i} F_{, j}}{F^{2}}-\frac{1}{4} \frac{F_{, i} J_{, j}}{F J}-\frac{1}{4} \frac{F_{, j} J_{, i}}{F J}+\frac{J_{, i j}}{2 J}-\frac{3}{4} \frac{J_{, i} J_{, j}}{J^{2}}=-\kappa_{5} \varphi_{, i} \varphi_{, j} \quad \text { for } \quad i \neq j .
\end{aligned}
$$

\section{5D GAUGE INVARIANT METRIC FLUCTUATIONS ON COSMOLOGICAL SCALES}

On cosmological scales the next condition is satisfied

$$
\frac{G \zeta \psi_{0}}{2 a(\tau) r_{H}} \ll 1
$$

where $r_{H}$ denotes the value of the radial coordinate at the horizon entry. This means that on cosmological scales the functions $F(\tau, r)$ and $J(\tau, r)$ become independent of spatial coordinates [see eqs. in (4)], so that

$$
\left.F(\tau, r)\right|_{\frac{G \xi \psi_{0}}{2 a(\tau) r_{H}} \ll 1} \rightarrow a^{2}(\tau),\left.\quad J(\tau, r)\right|_{\frac{G \xi \psi_{0}}{2 a(\tau) r_{H}} \ll 1} \rightarrow a^{2}(\tau),
$$

and the metric (3) describes an universe which is nearly 3D spatially homogeneous and isotropic.

The perturbed field equations (11)-(13) with the condition (18), give us the diagonal Einstein equations on cosmological scales

$$
\begin{array}{r}
3 \mathcal{H}^{2}-6 \mathcal{H} \dot{\Phi}+3 \dot{\Phi}^{2}-\left[(\vec{\nabla} \Phi)^{2}-2 \nabla^{2} \Phi\right] e^{4 \Phi}-3 a^{2}\left[\frac{1}{\psi_{0}^{2}}-\left(\frac{\psi}{\psi_{0}}\right)^{2}\left(\stackrel{\star \star}{\Phi}-2 \stackrel{\star}{\Phi}^{2}\right)-\frac{4 \psi}{\psi_{0}^{2}} \stackrel{\star}{\Phi}\right] e^{2 \Phi} \\
=\frac{\kappa_{5}}{2}\left[\dot{\varphi}^{2}+e^{4 \Phi}(\vec{\nabla} \varphi)^{2}+\left(\frac{\psi}{\psi_{0}}\right)^{2} a^{2} e^{2 \Phi} \stackrel{\star}{\varphi}^{2}\right], \\
-\mathcal{H}^{2}-2 \dot{\mathcal{H}}+2 \ddot{\Phi}+6 \mathcal{H} \dot{\Phi}-5 \dot{\Phi}^{2}+\frac{1}{3} e^{4 \Phi}(\vec{\nabla} \Phi)^{2}+a^{2}\left[\frac{3}{\psi_{0}^{2}}+2\left(\frac{\psi}{\psi_{0}}\right)^{2} \stackrel{\star}{\Phi}^{2}-\frac{4 \psi}{\psi_{0}^{2}} \stackrel{\star}{\Phi}-\left(\frac{\psi}{\psi_{0}}\right)^{2} \stackrel{\star \star}{\Phi}\right] e^{2 \Phi} \\
=\frac{\kappa_{5}}{2}\left[\dot{\varphi}^{2}-\frac{1}{3} e^{4 \Phi}(\vec{\nabla} \varphi)^{2}-\left(\frac{\psi}{\psi_{0}}\right)^{2} a^{2} e^{2 \Phi} \stackrel{\star^{2}}{\varphi}\right], \\
-3\left(\mathcal{H}^{2}+\dot{\mathcal{H}}\right)+3\left(\ddot{\Phi}+4 \mathcal{H} \dot{\Phi}-3 \dot{\Phi}^{2}\right)+\left[(\vec{\nabla} \Phi)^{2}-\nabla^{2} \Phi\right] e^{4 \Phi}+\frac{6 a^{2}}{\psi_{0}^{2}}(1-\psi \stackrel{\star}{\Phi}) e^{2 \Phi} \\
=\frac{\kappa_{5}}{2}\left[\dot{\varphi}^{2}-e^{4 \Phi}(\nabla \varphi)^{2}+\left(\frac{\psi}{\psi_{0}}\right)^{2} a^{2} e^{2 \Phi} \star^{\star^{2}}\right],
\end{array}
$$

whereas the non-diagonal equations (14) to (17) become

$$
\begin{aligned}
-2 \Phi_{, i} \dot{\Phi}+2 \mathcal{H} \Phi_{, i}+2 \dot{\Phi}_{, i} & =\kappa_{5} \dot{\varphi} \varphi, i, \\
-6 \stackrel{\star}{\Phi} \dot{\Phi}+6 \mathcal{H} \stackrel{\star}{\Phi}+3 \stackrel{\star \star}{\Phi} & =\kappa_{5} \dot{\varphi} \dot{\varphi}^{\star}, \\
-2 \Phi_{, i} \stackrel{\star}{\Phi}+\stackrel{\star}{\Phi}_{, i} & =\kappa_{5} \stackrel{\star}{\varphi}, \dot{\varphi}, \\
-2 \Phi_{, i} \Phi_{, j} & =\kappa_{5} \varphi, i \varphi, \quad \text { for } \quad i \neq j .
\end{aligned}
$$


Here, $\mathcal{H}=\dot{a}(\tau) / a(\tau)$ is the Hubble parameter in conformal time. From the linear combination: [eq(22)$\mathrm{eq}(21)+(1 / 4) \times \mathrm{eq}(20)]$, results the expression

$$
\begin{array}{r}
-\frac{5}{4} \mathcal{H}^{2}-\dot{\mathcal{H}}+\ddot{\Phi}+\frac{9}{2} \mathcal{H} \dot{\Phi}-\frac{13}{4} \dot{\Phi}^{2}+\frac{5}{12}(\vec{\nabla} \Phi)^{2} e^{4 \Phi}-\frac{1}{2}\left(\nabla^{2} \Phi\right) e^{4 \Phi}+a^{2}\left[\frac{9}{4 \psi_{0}^{2}}-\frac{7}{2}\left(\frac{\psi}{\psi_{0}}\right)^{2}\left(\stackrel{\star \star}{\Phi}^{2}-\frac{1}{2} \stackrel{\star \star}{\Phi}\right)+\frac{\psi}{\psi_{0}^{2}} \stackrel{\star}{\Phi}\right] e^{2 \Phi} \\
=\frac{\kappa_{5}}{2}\left[\frac{1}{4} \dot{\varphi}^{2}-\frac{5}{12} e^{4 \Phi}(\vec{\nabla} \varphi)^{2}+\frac{9}{4}\left(\frac{\psi}{\psi_{0}}\right)^{2} a^{2} e^{2 \Phi} \stackrel{\star}{\varphi}^{2}\right]
\end{array}
$$

This equation describes the dynamics of the $5 \mathrm{D}$ scalar metric fluctuations $\Phi(\tau, x, y, z, \psi)$ in terms of the $5 \mathrm{D}$ scalar field $\varphi(\tau, x, y, z, \psi)$, which in $4 \mathrm{D}$ will be formally identified with the inflaton field. Finally, the equation (10) for $\varphi(\tau, x, y, z, \psi)$ yields

$$
\ddot{\varphi}+(2 \mathcal{H}-4 \dot{\Phi}) \dot{\varphi}-e^{4 \Phi} \nabla^{2} \varphi+\left(\frac{\psi}{\psi_{0}}\right)^{2} a^{2} e^{2 \Phi}\left[\left(2 \stackrel{\star}{\Phi}-\frac{4}{\psi}\right) \stackrel{\star}{\varphi}-\stackrel{\star \star}{\varphi}\right]=0
$$

which is the equation that describes the 5D dynamics of the scalar field $\varphi$ on cosmological scales in presence of the scalar metric fluctuations.

\section{WEAK FIELD LIMIT OF 5D METRIC FLUCTUATIONS}

In the previous section we have derived the $5 \mathrm{D}$ field equations describing the dynamics of the $5 \mathrm{D}$ gauge-invariant scalar metric fluctuations $\Phi$, in a non-perturbative manner. The amplitudes of these metric fluctuations have no restrictions neither on cosmological or astrophysical scales. However, as it is well-known, such amplitudes are small on cosmological scales and specially during an inflationary stage of the early universe. Due to this fact we shall consider small amplitudes, in order to study the spectrum of these scalar metric fluctuations during inflation on large-scales. We shall consider the weak field limit approximation: $e^{ \pm 2 \Phi} \simeq 1 \pm 2 \Phi$.

Let us start with the weak field limit of the equation (28). Linearizing this equation respect to $\Phi$ we obtain

$$
\ddot{\varphi}-(2 \mathcal{H}-4 \dot{\Phi}) \dot{\varphi}-(1+4 \Phi) \nabla^{2} \varphi+\left(\frac{\psi}{\psi_{0}}\right)^{2} a^{2}\left[\left(2 \stackrel{\star}{\Phi}-\frac{4}{\psi}\right) \stackrel{\star}{\varphi}-(1+2 \Phi) \stackrel{\star \star}{\varphi}-\frac{8}{\psi} \Phi^{\star} \stackrel{\varphi}{\varphi}\right]=0 .
$$

Now, implementing a semi-classical approximation for the scalar field: $\varphi(\tau, x, y, z, \psi)=\varphi_{b}(\tau, \psi)+\delta \varphi(\tau, x, y, z, \psi)$, in the equation (29), we obtain separately the dynamics for both, the background part of the field $\varphi_{b}$ and for the quantum fluctuations $\delta \varphi$

$$
\begin{aligned}
& \ddot{\varphi}_{b}+2 \mathcal{H} \dot{\varphi}_{b}-\left(\frac{\psi}{\psi_{0}}\right)^{2} a^{2}\left[\frac{4}{\psi} \stackrel{\star}{\varphi}_{b}+\stackrel{\star \star}{\varphi}_{b}\right]=0, \\
& \ddot{\delta} \varphi+2 \mathcal{H} \dot{\delta} \dot{\varphi}-\nabla^{2} \delta \varphi-\left(\frac{\psi}{\psi_{0}}\right)^{2} a^{2}\left[\frac{4}{\psi} \hat{\delta}^{\star} \varphi+\stackrel{\star \star}{\delta} \varphi\right]-4 \dot{\varphi}_{b} \dot{\Phi}+\left(\frac{\psi}{\psi_{0}}\right)^{2} a^{2}\left[2 \stackrel{\star}{\varphi}_{b}^{\star} \stackrel{\star}{\Phi}-\left(\frac{8}{\psi} \stackrel{\star}{\varphi}_{b}+2 \stackrel{\star \star}{\varphi}_{b}\right) \Phi\right]=0 .
\end{aligned}
$$

Similarly, linearizing with respect to $\Phi$ the equations (20) to (22), we obtain for the background field $\varphi_{b}$

$$
\begin{array}{r}
3 \mathcal{H}^{2}-\frac{3 a^{2}}{\psi_{0}^{2}}=\frac{\kappa_{5}}{2}\left[\dot{\varphi}_{b}^{2}+\left(\frac{\psi}{\psi_{0}}\right)^{2} a^{2} \stackrel{\star}{\varphi}_{b}^{2}\right], \\
-\mathcal{H}^{2}-2 \dot{\mathcal{H}}+\frac{3 a^{2}}{\psi_{0}^{2}}=\frac{\kappa_{5}}{2}\left[\dot{\varphi}_{b}^{2}-\left(\frac{\psi}{\psi_{0}}\right)^{2} a^{2} \stackrel{\star}{\varphi}_{b}^{2}\right], \\
-3\left(\mathcal{H}^{2}+\dot{\mathcal{H}}\right)+\frac{6 a^{2}}{\psi_{0}^{2}}=\frac{\kappa_{5}}{2}\left[\dot{\varphi}_{b}^{2}+\left(\frac{\psi}{\psi_{0}}\right)^{2} a^{2} \stackrel{\star}{\varphi}_{b}^{2}\right],
\end{array}
$$


while that for the field $\delta \varphi$, we have

$$
\begin{aligned}
& -6 \mathcal{H} \dot{\Phi}+2 \nabla^{2} \Phi+3 a^{2}\left[\left(\frac{\psi}{\psi_{0}}\right)^{2} \stackrel{\star \star}{\Phi}+\frac{4 \psi}{\psi_{0}^{2}} \stackrel{\star}{\Phi}-\frac{2}{\psi_{0}^{2}} \Phi\right]=\kappa_{5}\left[\dot{\varphi}_{b} \delta \dot{\varphi}+\left(\frac{\psi}{\psi_{0}}\right)^{2} a^{2}\left(\stackrel{\star}{\varphi}_{b} \delta^{\star} \varphi+\stackrel{\star}{\varphi}_{b}^{2} \Phi\right)\right], \\
& 2 \ddot{\Phi}+6 \mathcal{H} \dot{\Phi}+a^{2}\left[\frac{6}{\psi_{0}^{2}} \Phi-\left(\frac{\psi}{\psi_{0}}\right)^{2} \stackrel{\star \star}{\Phi}-\frac{4 \psi}{\psi_{0}^{2}} \stackrel{\star}{\Phi}\right]=\kappa_{5}\left[\dot{\varphi}_{b} \dot{\delta} \varphi-\left(\frac{\psi}{\psi_{0}}\right)^{2} a^{2}\left(\stackrel{\star}{\varphi}_{b} \delta^{\star} \varphi+\star^{\star}{ }_{b} \Phi\right)\right], \\
& 3(\ddot{\Phi}+4 \mathcal{H} \dot{\Phi})-\nabla^{2} \Phi+\frac{6 a^{2}}{\psi_{0}^{2}}(2 \Phi-\psi \stackrel{\star}{\Phi})=\kappa_{5}\left[\dot{\varphi} b \dot{\delta} \varphi+\left(\frac{\psi}{\psi_{0}}\right)^{2} a^{2}\left(\stackrel{\star}{\varphi}_{b} \delta^{\star} \varphi+\stackrel{\star}{\varphi}_{b}^{2} \Phi\right)\right] .
\end{aligned}
$$

On the other hand, linearizing the non-diagonal field Einstein equations (23) to (26), we obtain

$$
\begin{aligned}
2\left(\mathcal{H} \Phi_{, i}+\dot{\Phi}_{, i}\right) & =\kappa_{5} \dot{\varphi}_{b} \delta \varphi_{, i} \\
6 \mathcal{H} \stackrel{\star}{\Phi}+3 \stackrel{\star \star}{\Phi} & =\kappa_{5}\left(\dot{\varphi}_{b} \delta^{\star} \varphi+\dot{\delta} \stackrel{\star}{\varphi}_{b}\right) \\
\stackrel{\star}{\Phi}_{, i} & =\kappa_{5} \stackrel{\star}{\varphi}_{b} \delta \varphi_{, i} .
\end{aligned}
$$

The equation (27) after be linearized can be written as

$$
-\frac{5}{4} \mathcal{H}^{2}-\dot{\mathcal{H}}+\frac{9 a^{2}}{4 \psi_{0}^{2}}=\frac{\kappa_{5}}{8}\left[\dot{\varphi}_{b}^{2}+9\left(\frac{\psi}{\psi_{0}}\right)^{2} a^{2} \stackrel{\star}{\varphi}_{b}^{2}\right]
$$

while that the dynamics of the field $\delta \varphi$, is given by

$$
\ddot{\Phi}+\frac{9}{2} \mathcal{H} \dot{\Phi}-\frac{1}{2} \nabla^{2} \Phi+a^{2}\left[\frac{9}{2 \psi_{0}^{2}} \Phi+\frac{\psi}{\psi_{0}^{2}} \stackrel{\star}{\Phi}+\frac{7}{4}\left(\frac{\psi}{\psi_{0}}\right)^{2} \stackrel{\star \star}{\Phi}\right]=\frac{\kappa_{5}}{4}\left[\dot{\varphi} \dot{\varphi} \dot{\varphi}+9\left(\frac{\psi}{\psi_{0}}\right)^{2} a^{2}\left(\stackrel{\star}{\varphi}_{b} \delta^{\star} \varphi+\stackrel{\star}{\varphi}_{b}^{2} \Phi\right)\right]
$$

This last equation describes the 5D dynamics of the metric fluctuations $\Phi$ in terms of the scalar field fluctuations $\delta \varphi$. Clearly, this equation can be also derived as a linear combination of the equations (35)-(37).

\section{INDUCING THE 4D DYNAMICS FOR THE NON-PERTURBATIVE GAUGE INVARIANT SCALAR METRIC FLUCTUATIONS}

Since we know the 5D field equations of motion for both, the scalar field $\varphi$ and the non-perturbative scalar metric fluctuations $\Phi$, we are in position to derive their respective dynamics on our 4D universe. With this in mind, we shall assume that the 5D spacetime can be foliated by a family of hypersurfaces $\Sigma: \psi=$ constant. Our $4 \mathrm{D}$ universe will be here represented by a generic hypersurface $\Sigma_{0}: \psi=\psi_{0}$. Thus, on every leaf member of the family, the line element induced by (3), has the form

$$
d s_{4}^{2}=F(\tau, r) d \tau^{2}-J(\tau, r)\left[d r^{2}+r^{2}\left(d \theta^{2}+\sin ^{2} \theta d \phi^{2}\right)\right]
$$

with

$$
F(\tau, r)=a^{2}(\tau)\left[1-\frac{G m}{2 a r}\right]^{2}\left[1+\frac{G m}{2 a r}\right]^{-2}, \quad J(\tau, r)=a^{2}(\tau)\left[1+\frac{G m}{2 a r}\right]^{4},
$$

where $m=\zeta \psi_{0}$ is the physical mass of a primordial black-hole which evolves during inflation and has a Schwarzschild radius $R_{H}=2 G m$. It is clear that the condition (18) delimits the cosmological scales. On the 4D hypersurface, this condition takes the form

$$
\frac{G m}{2 a r} \ll 1
$$

The background metric in (43) describes on cosmological scales a de-Sitter expansion of the early universe characterized by an equation of state $p_{b}=-\rho_{b}=-3 /\left(8 \pi G \psi_{0}^{2}\right)$, where $\rho_{b}$ and $p_{b}$ are the background energy density and the pressure. 
The 5D perturbed line element in cartesian coordinates (7), induces on the hypersurface $\Sigma_{0}$ the effective $4 \mathrm{D}$ line element

$$
\left.d s_{4}^{2}\right|_{\text {pert }}=F(\tau, \bar{x}) e^{2 \Omega(\tau, \bar{x})} d \tau^{2}-J(\tau, \bar{x}) e^{-2 \Omega(\tau, \bar{x})} \delta_{i j} d x^{i} d x^{j},
$$

where $\Omega(\tau, \bar{x}) \equiv \Phi\left(\tau, x, y, z, \psi_{0}\right)$ describes the 4 D scalar metric fluctuations induced on $\Sigma_{0}$. The equations (20)-(22) induce on our $4 \mathrm{D}$ spacetime $\Sigma_{0}$, the field equations

$$
\begin{aligned}
& 3 \mathcal{H}^{2}-6 \mathcal{H} \dot{\Omega}+3 \dot{\Omega}^{2}-\left[(\vec{\nabla} \Omega)^{2}-2 \nabla^{2} \Omega\right] e^{4 \Omega}-\left.3 a^{2} e^{2 \Omega}\left[\frac{1}{\psi_{0}^{2}}-\left(\frac{\psi}{\psi_{0}}\right)^{2}\left(\stackrel{\star \star}{\Phi}-2 \stackrel{\star}{\Phi}^{2}\right)-\frac{4 \psi}{\psi_{0}^{2}} \stackrel{\star}{\Phi}\right]\right|_{\psi=\psi_{0}} \\
& =4 \pi G\left[\dot{\bar{\varphi}}^{2}+e^{4 \Omega}(\vec{\nabla} \bar{\varphi})^{2}+\left.a^{2} e^{2 \Omega}\left(\frac{\psi^{2}}{\psi_{0}^{2}} \stackrel{\star}{\varphi}^{2}\right)\right|_{\psi=\psi_{0}}\right] \text {, } \\
& -\mathcal{H}^{2}-2 \dot{\mathcal{H}}+2 \ddot{\Omega}+6 \mathcal{H} \dot{\Omega}-5 \dot{\Omega}^{2}+\frac{1}{3} e^{4 \Omega}(\vec{\nabla} \Omega)^{2}+\left.a^{2} e^{2 \Omega}\left[\frac{3}{\psi_{0}^{2}}+2\left(\frac{\psi}{\psi_{0}}\right)^{2} \stackrel{\star}{\Phi}-4 \frac{\psi}{\psi_{0}^{2}} \stackrel{\star}{\Phi}-\left(\frac{\psi}{\psi_{0}}\right)^{2} \stackrel{\star \star}{\Phi}\right]\right|_{\psi=\psi_{0}} \\
& =4 \pi G\left[\dot{\bar{\varphi}}^{2}-\frac{1}{3} e^{4 \Omega}(\vec{\nabla} \bar{\varphi})^{2}-\left.a^{2} e^{2 \Omega}\left(\frac{\psi^{2} \star^{2}}{\psi_{0}^{2}}\right)\right|_{\psi=\psi_{0}}\right] \text {, } \\
& -3\left(\mathcal{H}^{2}+\dot{\mathcal{H}}\right)+3\left(\ddot{\Omega}+4 \mathcal{H} \dot{\Omega}^{2}-3 \dot{\Omega}^{2}\right)+\left[(\vec{\nabla} \Omega)^{2}-\nabla^{2} \Omega\right] e^{4 \Omega}+\left.6 a^{2} e^{2 \Omega}\left[\frac{1}{\psi_{0}^{2}}-\frac{\psi}{\psi_{0}^{2}} \stackrel{\star}{\Phi}\right]\right|_{\psi=\psi_{0}} \\
& =4 \pi G\left[\dot{\bar{\varphi}}^{2}-e^{4 \Omega}(\vec{\nabla} \bar{\varphi})^{2}+\left.a^{2} e^{2 \Omega}\left(\frac{\psi^{2} \star^{2}}{\psi_{0}^{2}}\right)\right|_{\psi=\psi_{0}}\right]
\end{aligned}
$$

where $\bar{\varphi}(\tau, x, y, z)=\left.\varphi(\tau, x, y, z, \psi)\right|_{\psi=\psi_{0}}$ is the massive scalar field induced on $\Sigma_{0}$. The dynamics of the induced scalar metric fluctuations $\Omega$, derived from (27) is given by

$$
\begin{aligned}
& -\frac{5}{4} \mathcal{H}^{2}-\dot{\mathcal{H}}+\ddot{\Omega}+\frac{9}{2} \mathcal{H} \dot{\Omega}-\frac{13}{4} \dot{\Omega}^{2}+\frac{5}{12}(\vec{\nabla} \Omega)^{2} e^{4 \Omega}-\frac{1}{2}\left(\nabla^{2} \Omega\right) e^{4 \Omega} \\
& +\left.a^{2} e^{2 \Omega}\left[\frac{9}{4 \psi_{0}^{2}}-\frac{7}{2}\left(\frac{\psi}{\psi_{0}}\right)^{2} \stackrel{\star^{2}}{\Phi}+\frac{\psi}{\psi_{0}^{2}} \stackrel{\star}{\Phi}+\frac{7}{4}\left(\frac{\psi}{\psi_{0}}\right)^{2} \stackrel{\star \star}{\Phi}\right]\right|_{\psi=\psi_{0}} \\
& =\frac{8 \pi G}{2}\left[\frac{1}{4} \dot{\bar{\varphi}}^{2}-\frac{5}{12} e^{4 \Omega}(\vec{\nabla} \bar{\varphi})^{2}+\left.\frac{9}{4} a^{2} e^{2 \Omega}\left(\frac{\psi^{2}}{\psi_{0}^{2}} \stackrel{\star}{\varphi}^{2}\right)\right|_{\psi=\psi_{0}}\right],
\end{aligned}
$$

and the induced dynamics of $\bar{\varphi}$ on the hypersurface $4 \mathrm{D}$ is given by the equation

$$
\ddot{\bar{\varphi}}+[2 \mathcal{H}-4 \dot{\Omega}] \dot{\bar{\varphi}}-e^{4 \Omega} \nabla^{2} \bar{\varphi}+\left.a e^{2 \Omega}\left[\left(2 \stackrel{\star}{\Phi}-\frac{4}{\psi}\right) \stackrel{\star}{\varphi}-\stackrel{\star \star}{\varphi}\right]\right|_{\psi=\psi_{0}}=0
$$

which is derived by evaluating $(28)$ on $\Sigma_{0}$.

The $5 \mathrm{D}$ action (8) induces on our $4 \mathrm{D}$ spacetime the effective action

$$
{ }^{(4)} \mathcal{S}_{e f f}=\int d^{4} x \sqrt{g_{4}}\left[\frac{{ }^{(4)} R}{16 \pi G}-\frac{1}{2} g^{\mu \nu} \bar{\varphi}_{, \mu} \bar{\varphi}_{, \nu}+V(\bar{\varphi})\right],
$$

where $g_{4}$ is the determinant of the $4 \mathrm{D}$ induced metric, which for the background reads $g_{4}^{(b)}=F J^{3}$ while for the perturbed metric $g_{4}^{(p)}=F J^{3} e^{-4 \Omega}$. The $4 \mathrm{D}$ Ricci scalar curvature ${ }^{(4)} R$ is given by

$$
{ }^{(4)} R=\frac{2}{a^{2}}\left[3\left(\mathcal{H}^{2}+\dot{\mathcal{H}}-4 \mathcal{H} \dot{\Omega}-\ddot{\Omega}+3 \dot{\Omega}^{2}\right) e^{-2 \Omega}+\left(\nabla^{2} \Omega-(\nabla \Omega)^{2}\right) e^{2 \Omega}\right],
$$

and the induced $4 \mathrm{D}$ effective potential $V$ has the form

$$
V(\bar{\varphi})=-\left.\frac{1}{2} g^{\psi \psi}\left(\frac{\partial \varphi}{\partial \psi}\right)^{2}\right|_{\psi=\psi_{0}}
$$


In our analysis the fields $\Omega$ and $\bar{\varphi}$ are semi-classical fields, so they are constituted by a classical part plus a quantum part. To study the dynamics of the former, a standard quantization procedure will be implemented. To do it, we shall impose the commutation relations

$$
\left[\bar{\varphi}(\tau, \bar{x}), \Pi_{(\bar{\varphi})}^{0}\left(\tau, \bar{x}^{\prime}\right)\right]=i \delta^{(3)}\left(\bar{x}-\bar{x}^{\prime}\right), \quad\left[\Omega(\tau, \bar{x}), \Pi_{(\Omega)}^{0}\left(\tau, \bar{x}^{\prime}\right)\right]=i \delta^{(3)}\left(\bar{x}-\bar{x}^{\prime}\right),
$$

where $\bar{x}$ is denoting the $3 \mathrm{D}$ vector position in cartesian coordinates. Due to the fact that the conjugate momentum to $\bar{\varphi}$ and $\Omega$, are respectively given by $\Pi_{(\bar{\varphi})}^{0}=\sqrt{-g_{4}} F^{-1} e^{-2 \Omega} \dot{\bar{\varphi}}$ and $\Pi_{(\Omega)}^{0}=[12 /(16 \pi G)] a^{-2}(3 \dot{\Omega}-2 \mathcal{H}) e^{-2 \Omega} \sqrt{-g_{4}}$, the expressions (55) yield

$$
\left[\bar{\varphi}(\tau, \bar{x}), \dot{\bar{\varphi}}\left(\tau, \bar{x}^{\prime}\right)\right]=\frac{i e^{2 \Omega}}{F \sqrt{-g_{4}}} \delta^{(3)}\left(\bar{x}-\bar{x}^{\prime}\right), \quad\left[\Omega(\tau, \bar{x}), \dot{\Omega}\left(\tau, \bar{x}^{\prime}\right)\right]=i \frac{4 \pi G a^{2} e^{2 \Omega}}{9 \sqrt{-g_{4}}} \delta^{(3)}\left(\bar{x}-\bar{x}^{\prime}\right) .
$$

\section{INDUCED 4D DYNAMICS FOR THE INFLATON AND GAUGE INVARIANT METRIC FLUCTUATIONS IN THE WEAK FIELD LIMIT}

In the section IV we have obtained the weak field limit of the 5D field equations for both fields: the inflaton field $\varphi$ and the scale invariant metric fluctuation field $\Phi$. A similar mechanism can be applied in the case of the effective 4D field equations of the section V. Thus, let us use the semiclassical approximation: $\bar{\varphi}(\tau, \bar{r})=\bar{\varphi}_{b}(\tau)+\delta \bar{\varphi}(\tau, \vec{r})$, where $\left.\bar{\varphi}_{b}(\tau) \equiv \bar{\varphi}_{b}(\tau, \psi)\right|_{\psi=\psi_{0}}$ is the background 4D inflaton field and $\left.\delta \bar{\varphi}(\tau, \vec{r}) \equiv \delta \bar{\varphi}(\tau, \vec{r}, \psi)\right|_{\psi=\psi_{0}}$ stands for the 4D inflaton field quantum fluctuations. Taking this into account, the evaluation of equation (30) on $\Sigma_{0}$ yields

$$
\ddot{\bar{\varphi}}_{b}+2 \mathcal{H} \dot{\bar{\varphi}}_{b}+a^{2} m^{2} \bar{\varphi}_{b}=0
$$

where we have used the relation: $\left.\left[(4 / \psi) \stackrel{\star}{\varphi}_{b}+\stackrel{\star \star \star}{\varphi}_{b}\right]\right|_{\psi=\psi_{0}}=-m^{2} \bar{\varphi}_{b}$, such that $m$ is a separation constant. Now, according to the equation (47), the background field $\bar{\varphi}$ must obey the Friedmann-like equation

$$
\left(\frac{\partial \bar{\varphi}_{b}}{\partial \tau}\right)^{2}+a^{2}\left(\frac{\partial \varphi_{b}}{\partial \psi}\right)_{\psi=\psi_{0}}^{2}=0
$$

A particular solution of (57), that also is satisfied when inflation begins, are the slow rolling conditions: $\partial \bar{\varphi}_{b} / \partial \tau=0$, where necessarily $m=0$ [12]. Using this solution in (58), it yields

$$
\bar{\varphi}_{b}=0
$$

which means that all the energy density on the 4D hypersurface is induced geometrically by the foliation $\psi=\psi_{0}=1 / H$, because the background energy density related to the background inflaton field is null.

On the other hand, by using (39), (40) and (42), we obtain after some algebra that the scalar metric fluctuations $\Omega$ on $\Sigma_{0}$ obey the equation

$$
\ddot{\Omega}+\frac{9}{2} \mathcal{H} \dot{\Omega}-\frac{1}{2} \nabla^{2} \Omega+\left[\frac{9}{2} a^{2} H^{2}+\lambda^{2}\right] \Omega=0 .
$$

Here, we have used $\left\{\left(\psi / \psi_{0}^{2}-9 / \psi\right) \stackrel{\star}{\Phi}+\left[(7 / 4)\left(\psi / \psi_{0}\right)^{2}-9 / 4\right]^{\star \star}\right\}_{\psi=\psi_{0}}=\lambda^{2} \Omega$, where $\lambda$ is a separation constant with mass units. Now can be shown that the equation (38), evaluated on $\Sigma_{0}$, leaves to the condition $\dot{\Omega}=-\mathcal{H} \Omega$. Using this last condition in the equation (60), we obtain

$$
\ddot{\Omega}+2 \mathcal{H} \dot{\Omega}-\frac{1}{2} \nabla^{2} \Omega+\left[a^{2} H^{2}+\lambda^{2}\right] \Omega=0 .
$$

If we introduce the auxiliary field $\chi(\tau, \bar{r})$, through the formula

$$
\Omega(\tau, \bar{r})=e^{-\int \mathcal{H}(\tau) d \tau} \chi(\tau, \bar{r})
$$

the equation (61), becomes

$$
\ddot{\chi}-\frac{1}{2} \nabla^{2} \chi+\left(\lambda^{2}-\dot{\mathcal{H}}\right) \chi=0 .
$$


Now, in order to quantize the field $\chi$, we shall expand it in Fourier modes

$$
\chi(\tau, \bar{r})=\frac{1}{(2 \pi)^{3 / 2}} \int d^{3} k\left[a_{k} e^{i \bar{k} \cdot \bar{r}} \xi_{k}(\tau)+a_{k}^{\dagger} \xi_{k}^{*}(\tau)\right],
$$

where the annihilation and creation operators $a_{k}$ and $a_{k}^{\dagger}$, satisfy the commutation algebra

$$
\left[a_{k}, a_{k^{\prime}}^{\dagger}\right]=\delta^{(3)}\left(\bar{k}-\bar{k}^{\prime}\right), \quad\left[a_{k}, a_{k^{\prime}}\right]=\left[a_{k}^{\dagger}, a_{k^{\prime}}^{\dagger}\right]=0 .
$$

Inserting (64) in (63) and using (65), we obtain

$$
\ddot{\xi}_{k}+\left(k_{e f f}^{2}-\frac{2}{\tau^{2}}+\lambda^{2}\right) \xi_{k}=0,
$$

where $k_{\text {eff }}^{2}=k^{2} / 2$. Using (64) and (65) in the second expression of (56), the modes $\xi_{k}$ must satisfy in the UV-sector the normalization condition

$$
\xi_{k} \dot{\xi}_{k}^{*}-\xi_{k}^{*} \dot{\xi}_{k}=i \frac{4 \pi G}{9 a_{0}^{2}}
$$

Thus, choosing the Bunch-Davies vacuum condition, the normalized solution of (66) reads

$$
\xi_{k}(\tau) \simeq \frac{i \pi}{3 a_{0}} \sqrt{G} \mathcal{H}_{\nu}^{(2)}[z(\tau)]
$$

where $\mathcal{H}_{\nu}^{(2)}[z(\tau)]$ is the second kind Hankel function, $\nu=(1 / 2) \sqrt{1+4 \beta}$ and $z(\tau)=k_{\text {eff }} \tau$. Notice that $\beta=2-\lambda^{2} \tau^{2}>$ 0 , such that the conformal time at the beginning of inflation $\tau_{0}=-\frac{\sqrt{2}}{\lambda}$ is defined such that $\beta_{0}=2-\lambda^{2} \tau_{0}^{2}=0$ and the conformal time at the end of inflation complies with $\beta_{e}=2-\lambda^{2} \tau_{e}^{2} \simeq 2$. Using the definition of $\beta$, it is easy to show that the parameter $\nu \simeq 3 / 2$ for $\tau_{e}^{2} \ll 2 / \lambda^{2}$, which assures that the spectral index $1>n_{s}>0.96[13]$. Using the fact that $1-n_{s}=3-2 \nu$, we obtain that the range of acceptable values for $\tau_{e}$ is

$$
0<\left(-\tau_{e}\right)<\frac{0.245}{\lambda}
$$

Figure (1) shows the evolution of $n_{s}(\tau)$ during inflation for $\lambda=10^{-10} G^{-1 / 2}$. It is important to see how the universe becomes scale invariant with the expansion of the universe. Notice that the temporal evolution of $\beta$, and hence of the spectral index $n_{s}$, is due to the existence of $\lambda$, which has a clear origin in the extra space-like coordinate $\psi$ [see eqs. (60) and below]. This result cannot be found in standard 4D inflationary models.

\section{FINAL COMMENTS}

We have studied a non-perturbative formalism for scalar metric fluctuations from a 5D extended Schwarzschild-de Sitter (SdS) cosmological metric. In particular, we have studied the large-scale cosmological limit, which is the relevant for the study of large-scale structure formation in the early inflationary universe. In this limit the intensity of the fluctuations are weak enough, so we can take a longitudinal gauge, and thus one can implement the approximation $e^{ \pm 2 \Phi} \simeq 1 \pm 2 \Phi$. We have shown that the energy density on the $4 \mathrm{D}$ hypersurface is induced geometrically by the foliation $\psi=\psi_{0}=1 / H$, in virtue that the background energy density coming from the inflaton field is null. This is an important result because all the contributions of the inflaton field are in the quantum fluctuations. We have calculated the spectral index for the scale invariant metric fluctuations. Our results show how the universe becomes gradually scale invariant. The time when inflation ends $\tau_{e}$ can be calculated from experimental data. The range of possible values was obtained in (69). Finally, our 5D model resolves the problem of some 4D standard inflationary models in which the background values of the inflaton field $\bar{\varphi}_{b}$ are tansplanckians. In our case $\bar{\varphi}_{b}=0$, so that all the background energy density is due to the 4D induced cosmological constant, which is induced on the 4D hypersurface by the foliation $\psi=\psi_{0}=1 / H$.

\section{Acknowledgements}

J. E. Madriz-Aguilar, L. M. Reyes and C. Moreno acknowledge CONACYT (Mexico) and Mathematics Department of CUCEI- UdG for financial support. M. Bellini acknowledges CONICET (Argentina) and UNMdP for financial support. 


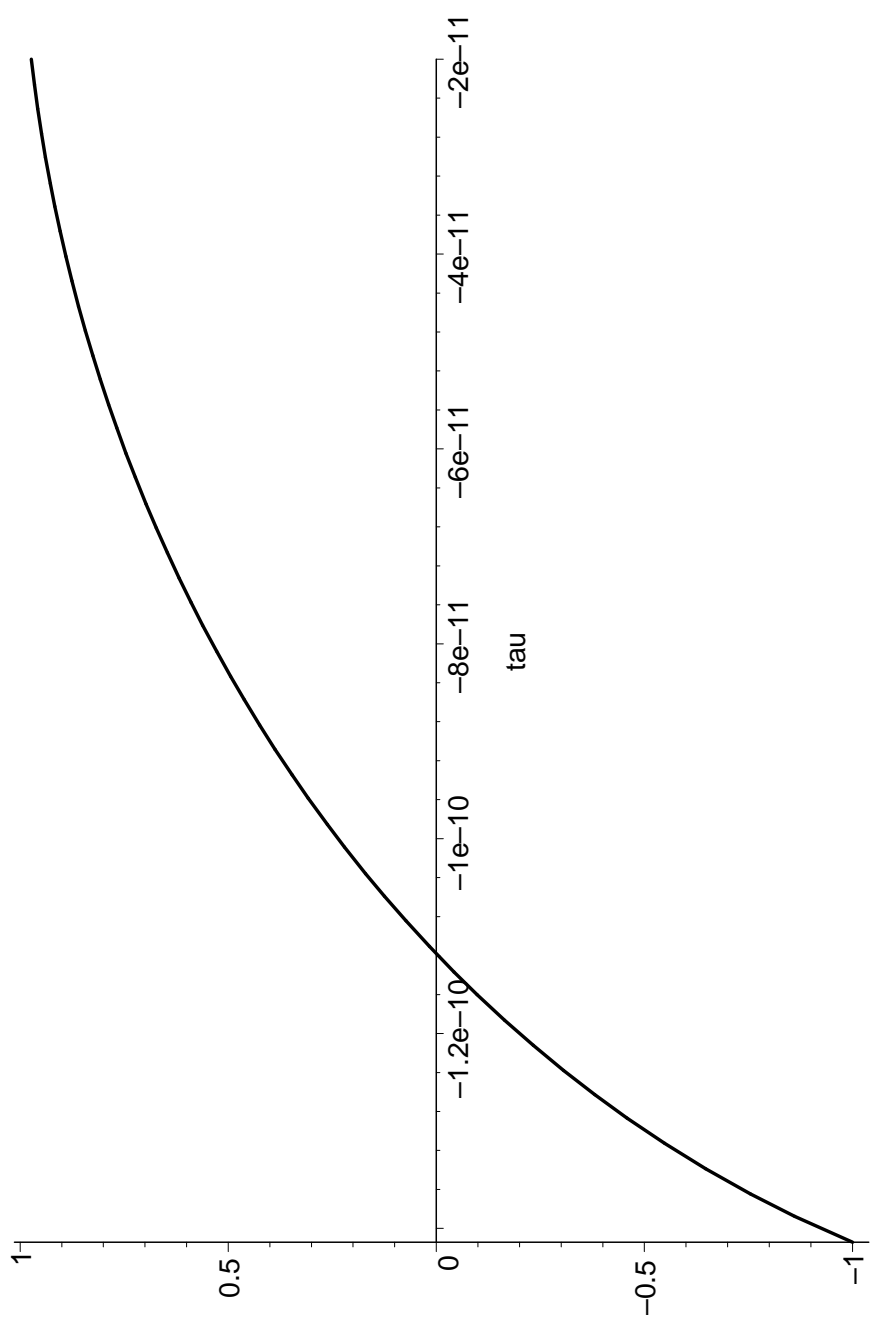

FIG. 1: Evolution of $n_{s}(\tau)$ during inflation for $\lambda=10^{-10} G^{-1 / 2}$. The universe becomes scale invariant with the growth of the universe.

[1] A. H. Guth, Phys. Rev. D23: 347(1981).

[2] D. H. Lyth and A. Riotto, Phys. Rept. 314: 1(1999).

[3] R. H. Brandenberger, Lec. Notes Phys. 738, (2008) 393-424.

[4] Kei-ichi Maeda, Nobuyoshi Ohta, Phys. Lett. B597 (2004) 400-407.

[5] P. S. Wesson. Gen. Rel. Grav. 35: 111(2003).

[6] J. E. Madriz Aguilar, M. Bellini, Phys. Lett. B679: 306(2009).

[7] J. E. Madriz Aguilar, M. Bellini, JCAP 1011: 020(2010);

L. M. Reyes, J. E. Madriz Aguilar, M. Bellini. Eur. Phys. J. Plus 126: 56(2011).

[8] L. M. Reyes, C. Moreno, J. E. Madriz Aguilar, M. Bellini. Phys. Lett. B717: 17(2012).

[9] Other approach for a SdS cosmological model of the early universe (but developed in a 4D model), can be seen in: Tomislav Prokopec, Paul Reska. JCAP 11: 050(2011).

[10] J. E. Madriz-Aguilar and M. Bellini, Phys. Lett. B679: 306(2009).

[11] T. Shiromizu, D. Ida and T. Torii, JHEP 11: 010(2001).

[12] E. J. Copeland, E. W. Kolb, A. R. Liddle and J. E. Lidsey, Phys. Rev. D 48: 2529(1993).

[13] O. Lahav and A. R. Liddle. Phys. Rev. D80: 280 (2012). 